\title{
Electronic Raman scattering through a stripe ordering transition in $\mathrm{La}_{2-x} \mathrm{Sr}_{x} \mathrm{NiO}_{4}$
}

\author{
V. P. Gnezdilov ${ }^{1}$, Yu. G. Pashkevich ${ }^{2}$, A. V. Yeremenko ${ }^{1}$, P. Lemmens ${ }^{3}$, \\ G. Güntherodt ${ }^{4}$, J. M. Tranquada ${ }^{4}$, D. J. Buttrey ${ }^{5}$, and K. Nakajima ${ }^{6}$ \\ ${ }^{1}$ B. Verkin Institute for Low Temperature Physics and Engineering of the National Academy of \\ Sciences of Ukraine, 47 Lenin Ave., Kharkov 61103, Ukraine \\ E-mail:gnezdilov@ilt.kharkov.ua
}

${ }^{2}$ A. Galkin Donetsk Physico-Technical Institute of the National Academy of Sciences of Ukraine, 72 R. Luxemburg Str., Donetsk 83114, Ukraine

${ }^{3}$ Physikalisches Institut, RWTH Aachen, Aachen 52056, Germany,

${ }^{4}$ Brookhaven National Laboratory, Upton, NY 11973,

${ }^{5}$ University of Delaware, Newark, Delaware 19716 ,

${ }^{6}$ Neutron Scattering Laboratory ISSP, University of Tokyo, Tokai, Ibaraki, Japan

Received February 28, 2002

\begin{abstract}
We describe the results of electronic Raman scattering experiments in two differently doped single crystals of $\mathrm{La}_{2}-x \mathrm{Sr}_{x} \mathrm{NiO}_{4}(x=0.225$ and $1 / 3)$. In $B_{1}$ symmetry a crossover from weakly interacting to pseudogap-like behavior is observed at a charge-ordering temperature $T_{c o}$. In $B_{2 g}$ symmetry a redistribution of electronic continua with decreasing temperature is accompanied by a loss of spectral weight below $T_{C O}$ in the low-frequency region due to opening of a pseudogap. The slope of the Raman response at vanishing frequencies is investigated, too. Its temperature behavior in $B_{2 g}$ symmetry, which predominantly selects charge carriers with momenta along the diagonals of the $\mathrm{NiO}_{2}$ bonds, provides clear evidence for one-dimensional charge transport in the charge-ordered phase.
\end{abstract}

PACS: 78.30.-j

\section{Introduction}

Stripe ordering of charge and spin in transition-metal oxides has been of intense interest to condensed-matter physics from the theoretical and experimental points of view as an example of a nontrivial ordering phenomenon that originates from the interplay between charge hybridization and interaction. The first evidence for unusual magnetic correlations was obtained in a neutron diffraction study on a single crystal of $\mathrm{La}_{1.8} \mathrm{Sr}_{0.2} \mathrm{NiO}_{3.96}$ [1]. Indications of charge order in $\mathrm{La}_{2-x} \mathrm{Sr}_{x} \mathrm{NiO}_{4}$ were found in electron diffraction [2] and transport measurements [3] on ceramic samples. Neutron diffraction studies $[4,5]$ of a $\mathrm{La}_{2} \mathrm{NiO}_{4.125}$ crystal were the first to detect diffraction from both the mag- netic and charge order in the same sample. In the first studies of $\mathrm{La}_{2-x} \mathrm{Sr}_{x} \mathrm{NiO}_{4}$ it has been suggested that ordering of the dopant-induced holes occurs only commensurately at special values of $x$, such as $1 / 2$ and $1 / 3[2,3]$. Later it was found that a single crystal with $x=0.2$, although not at a special value of $x$, shows commensurate order [6], albeit with a short in-plane correlation length of $\sim 40 \AA$. In contrast, the stripe order in $\mathrm{La}_{2} \mathrm{NiO}_{4+\delta}$ [4,5] and $\mathrm{La}_{1.775} \mathrm{Sr}_{0.225} \mathrm{NiO}_{4}$ [7] was found to be incommensurate, with the wave vector varying significantly with temperature.

The stripe order in Sr-doped nickelates has been characterized in detail by neutron diffraction, and some summary of the results is given in Ref. 8 . While many features of the ordering are now clear, 
some questions remain. One of them is the question of the possibility of finite conductivity in the stripe-ordered state. One can expect two possible scenarios, which could lead to conductivity. According to the first one the stripes themselves are insulating but the system can be metallic due to fluctuations and motion of stripes [9]. Alternatively, metallic conductivity may exist along the charge threads without a violation of stripe ordering as a whole. In the latter case, Coulomb interactions between neighboring stripes should lead to charge-density-wave order along stripes at sufficiently low temperatures and in the absence of stripe fluctuations [10]. In our previous optical conductivity study of a $\mathrm{La}_{1.775} \mathrm{Sr}_{0.225} \mathrm{NiO}_{4}$ single crystal a strong Fano antiresonance was observed in the optical conductivity spectra [11]. Based on a careful analysis of the phonon spectra, we concluded that the energy of the antiresonance corresponds to $\mathrm{Ni}-\mathrm{O}$ bond stretching motions along the stripes. It was concluded that the antiresonance, which results from electron-phonon coupling, provides strong evidence for finite conductivity along the stripes in the incommensurately stripe-ordered sample, at least at optical-phonon frequencies.

Raman scattering (RS) is a powerful method for studying the excitations of charge carriers in solids. In recent years this method has been widely applied to study the scattering of electrons in metals, insulators, semiconductors, and superconductors. Via light's coupling to the electron's charge, inelastic light scattering reveals symmetry-selective properties of the electron dynamics over a wide range of energy scales and temperatures. In this work we report on electronic Raman scattering spectra of two $\mathrm{La}_{2-x} \mathrm{Sr}_{x} \mathrm{NiO}_{4}$ single crystals with $x=0.225$ and $x=1 / 3$ and with charge ordering temperatures $T_{c o}=150 \mathrm{~K}$ and $240 \mathrm{~K}$, respectively [7,12]. The hole density per Ni site in the sample with $x=0.225$ is less than 1 (in contrast to $x=1 / 3$, where the density is exactly 1$)$. It is known that in-plane resistivity $\rho_{a b}$ of Sr-doped lantanium nickelates is doping-dependent: it increases with hole concentration decreasing. And independently of the doping level, the resistivity increases by several orders below $T_{c o}[3,13,14]$, which indicates quenching of the charge degrees of freedom due to the ordering. At first glance the increase of the resistivity below $T_{c o}$ is incompatible with possible conductivity along the threads of the stripes, which remain in the charge-ordered state. This contradiction can be removed by stripe domain formation, which occurs below $T_{c o}$. One can expect the appearance of two type thermodynamical stripe-domains in which stripes run perpendicular to each other. Thus, only small part of the threads of charges can participate in the charge transfer. Three principal symmetries $A_{1 g}$, $B_{1 g}$, and $B_{2 g}$, were examined. As has been described in detail in other publications [15-19], there exists a relationship between the charge-carrier momenta and light polarization through the symmetry properties of the Raman vertex. In $B_{1 g}$ and $B_{2 g}$ symmetry the charge carriers with momenta along the principal axes and the diagonals, respectively, are preferentially weighted. $A_{1 g}$ is a weighted average over the entire Brillouin zone.

\section{Experiment}

Raman spectra were measured on fresh chemically etched surfaces in a quasi-backscattering configuration utilizing a triple monochromator (DILOR XY), a liquid nitrogen cooled CCD detector, and a 514.5-nm Ar-ion laser. The laser beam of $20 \mathrm{~mW}$ was focused on an area of $0.1 \mathrm{~mm}^{2}$ on the $a b$ plane of the mirrorlike polished crystal surface. The orientation of the crystals in the $I 4 / \mathrm{mmm}$ setting was monitored by $\mathrm{x}$-ray Laue diffraction.

All measurements were performed with the polarization of incident and scattered light as $\left(\hat{\mathbf{E}}_{i}, \hat{\mathbf{E}}_{s}\right)=(x x),(x y)$, and $(a b)$, respectively. Here $a=[100]$ and $b=[010]$ are directions along the $\mathrm{Ni}-\mathrm{O}-\mathrm{Ni}$ bonds; the $x$ and $y$ directions are parallel to [110] and [1 $\overline{1} 0]$. Such geometries allow measuring the $A_{1 g}+B_{2 g}, B_{1 g}$, and $B_{2 g}$ symmetry components of the Raman-scattering cross section. The Raman response functions $\chi^{\prime \prime}(\omega)$ were obtained by dividing the original spectra $I(\omega, T)$ by Bose-Einstein thermal factor, since they are related to each other through $I(\omega, T) \sim\left[1-\exp \left(-\hbar \omega / k_{B}\right)\right]^{-1} \chi^{\prime \prime}(\omega, T)$.

\section{Results and discussion}

Raman scattering spectra of $\mathrm{La}_{2-x} \mathrm{Sr}_{x} \mathrm{NiO}_{4}$ $(x=1 / 3,0.225)$ and $\mathrm{La}_{2} \mathrm{NiO}_{4.125}$ in the $x x$ and $x y$ scattering geometries are presented elsewhere $[11,20-22]$. The average symmetry of the lattice is described by space group $I 4 / \mathrm{mmm}$. The corresponding Raman-active phonons are distributed among the irreducible representations of the space group as $2 A_{1 g}+2 E_{g}$. At room temperature all the observed modes are weak and broad. Conspicuous changes were observed in the phonon spectra below the charge-ordering temperature $T_{c o}$. The occurrence of stripe order, with a characteristic wave vector $\mathbf{Q}_{c}$, lowers the translational symmetry and leads to the appearance of extra lines both in the $x x$ and $x y$ spectra. Low-temperature scans in the $x y$ geometry reveal also two wide bands that were in- 
terpreted as two-magnon excitations within the antiferromagnetic domains and across the domain walls [11,20-22]. Phonon and two-magnon excitations are superposed on top of a significant electronic background that changes its shape with changing temperature.

As was noted in Ref. 23, RS experiments in strongly correlated systems (ranging from mixedvalence materials to Kondo insulators to high-temperature superconductors) show temperature-dependent electronic Raman spectra that are both remarkably similar and quite anomalous, suggesting a common mechanism governing transport. While theories that describe RS in weakly correlated metals [18] and band insulators [24] have been known for some time, a theory that connects the metallic and insulating states and describes materials near the metal-insulator transition has been developed only recently [23]. The theoretical model contains two types of electrons: itinerant band electrons and localized ( $d$ or $f$ ) electrons. The band electrons can hop between nearest neighbors (with hopping integral $t^{*} /(2 \sqrt{d})$ on a $d$-dimensional cubic lattice), and they interact via screened Coulomb interaction with the localized electrons (which is described by an interaction strength $U$ between electrons that are located at the same lattice site). The Hamiltonian is written as

$$
\begin{gathered}
H=-\frac{t^{*}}{2 \sqrt{d}} \sum_{(i, j)} d_{i}^{\dagger} d_{j}+E_{f} \sum_{i} w_{i}- \\
-\mu \sum_{i}\left(d_{i}^{\dagger} d_{i}+w_{i}\right)+U \sum_{i} d_{i}^{\dagger} d_{i} w_{i},
\end{gathered}
$$

where $d_{i}^{\dagger}\left(d_{i}\right)$ is the spinless conduction electron creation (annihilation) operator at lattice site $i$ and $w_{i}=0$ or 1 is a classical variable corresponding to the localized $f$-electron number at site $i$. Both $E_{f}$ and $\mu$ were adjusted so that the average filling of the $d$ electrons is $1 / 2$ and the average filling of the $f$ electrons is $1 / 2\left(\mu=U / 2\right.$ and $\left.E_{f}=0\right)$. For half-filling, $U<0.65$ corresponds to a weakly correlated metal, while a pseudogap phase appears for $0.65<U<1.5$, passing through a quantum critical point at $U=1.5$ to the insulator phase $U>1.5$ (the values of $U$ are presented in units of $\left.t^{*}\right)$.

Figures 1, $a$ and 2, $a$ show the $A_{1 g}$ experimental Raman spectra for the samples under study at different temperatures, that were obtained by subtracting the $(a b)$ spectra from the $(x x)$ ones. Figures $1, b$ and $2, b$ present the electronic Raman response for the $A_{1 g}$ channel evaluated at different temperatures by fitting the experimental curves

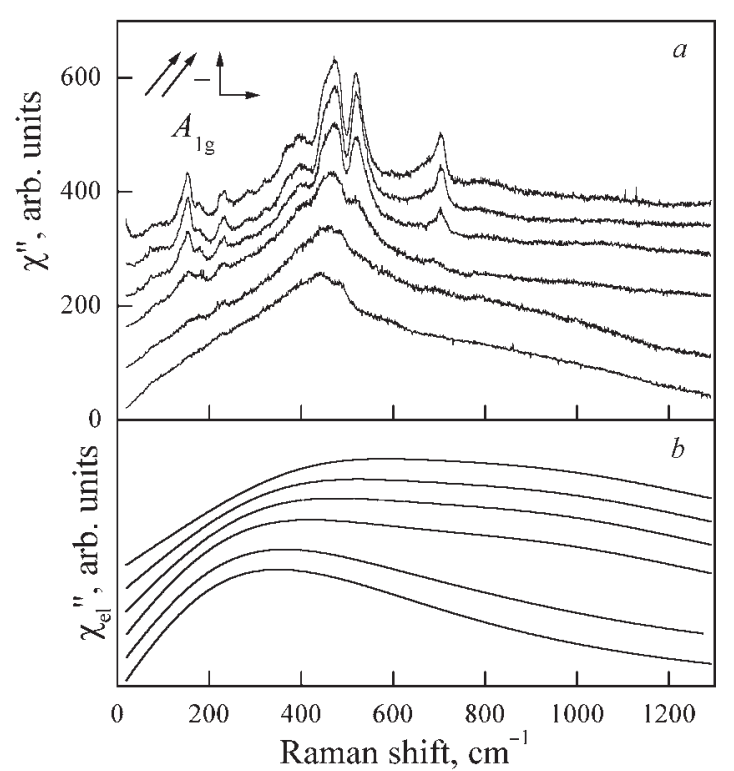

Fig. 1. Experimental $A_{1 g}$ Raman response of $\mathrm{La}_{1.775} \mathrm{Sr}_{0.225} \mathrm{NiO}_{4}$ at different temperatures $(T=5,50$, $100,150,200$, and $295 \mathrm{~K}$ from top to bottom). The spectra are plotted on the same scale but are displaced vertically for clarity ( $a$ ). Electronic $A_{1 g}$ Raman response at the same temperatures evaluated from the experimental spectra $(b)$.

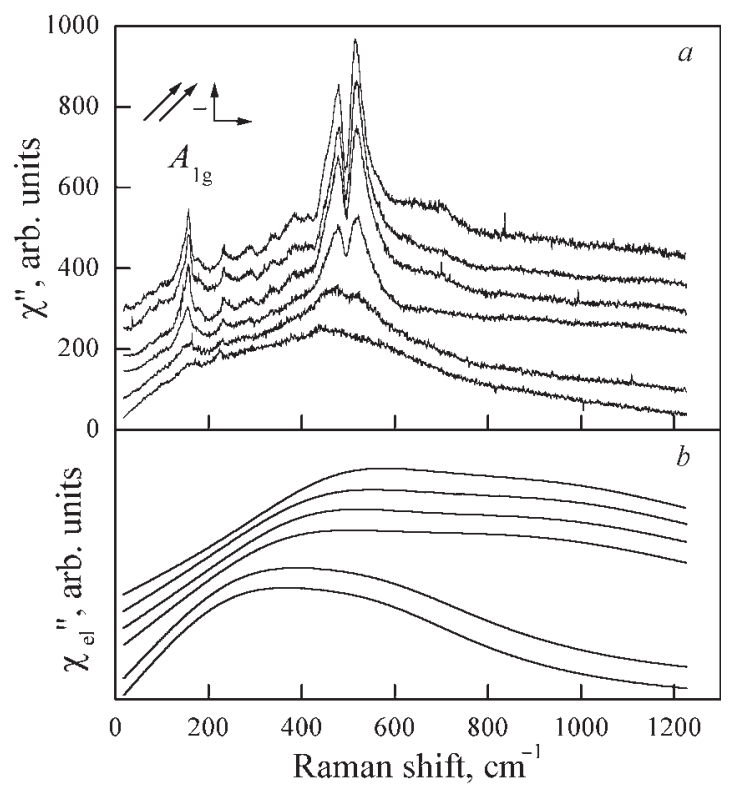

Fig. 2. Experimental $A_{1 g}$ Raman response of $\mathrm{La}_{5} /{ }_{3} \mathrm{Sr}_{1} /{ }_{3} \mathrm{NiO}_{4}$ at different temperatures $(T=5,50$, $100,150,250$, and $295 \mathrm{~K}$ from top to bottom). The spectra are plotted on the same scale but are displaced vertically for clarity $(a)$. Electronic $A_{1 g}$ Raman response at the same temperatures evaluated from the experimental spectra $(b)$.

and it is seen that the general behavior of the $A_{1 g}$ response is similar for both samples. As follows from the theory [23], the $A_{1 g}$ Raman response has a 


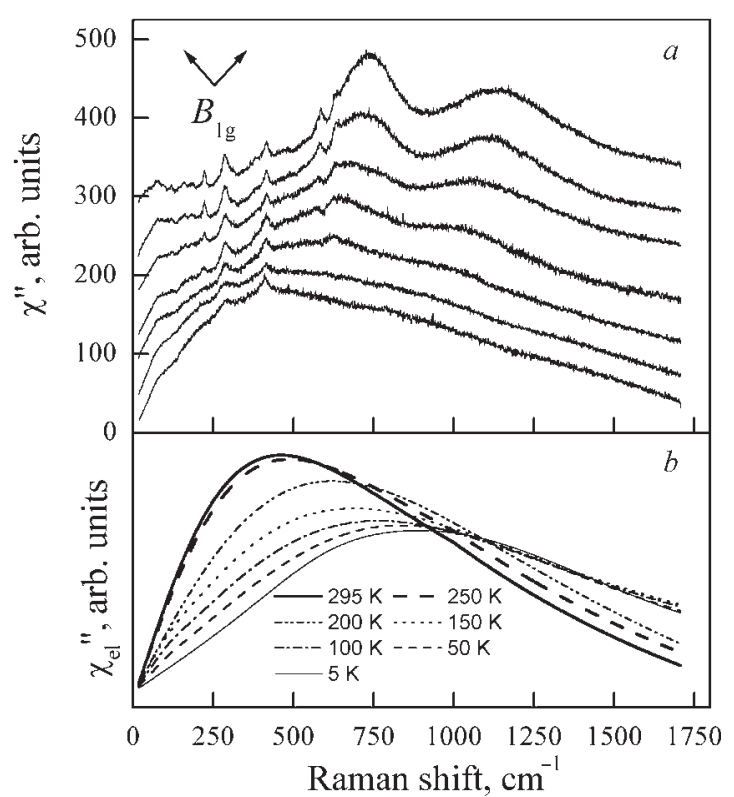

Fig. 3. Experimental $B_{1 g}$ Raman response of $\mathrm{La}_{1.775} \mathrm{Sr}_{0.225} \mathrm{NiO}_{4}$ at different temperatures $(T=5,50$, $100,150,200,250$, and $295 \mathrm{~K}$ from top to bottom). The spectra are plotted on the same scale but are displaced vertically for clarity $(a)$. Electronic $A_{1 g}$ Raman response at the same temperatures evaluated from the experimental spectra (b).

bell-like shape at all values of $U$ and increases and sharpens as $U$ increases. The peak of the response becomes more symmetric in shape and moves to higher energies also. Above $T_{c o}$, our samples display behavior consistent with the theory [23] and resistivity measurements [3,13,14]: (i) the Raman response function has an asymmetric line shape characteristic for $U<1.5$; (ii) the peak position shifts to lower energy with increasing hole concentration (decreasing resistivity).

Below $T_{c o}$, the $A_{1 g}$ Raman responses change to shapes composed of a rapidly increasing part from $\omega \sim 0$ to the leading edge energies and a weakly $\omega$-dependent part above them. The position of the leading edge depends on the temperature and shifts to higher energy as the temperature decreases.

In Figures 3, $a$ and 4, $a$ we plot $(x y)$ spectra versus temperature. Figures $3, b$ and $4, b$ present the electronic Raman response for the $B_{1 g}$ channel evaluated from the experimental spectra. In the charge-disordered state (above $T_{c o}$ ) the $B_{1 g}$ electronic Raman spectra for both samples are close to the ideal «bad metal» spectra. Note that the magnitude of resistivity above $T_{c o}$ corresponds to a «bad metal» also. As the temperature crosses $T_{c o}$, dramatic changes are observed in the spectra: the low-frequency response depletes and the spectral weight shifts into a charge-transfer peak. The posi-

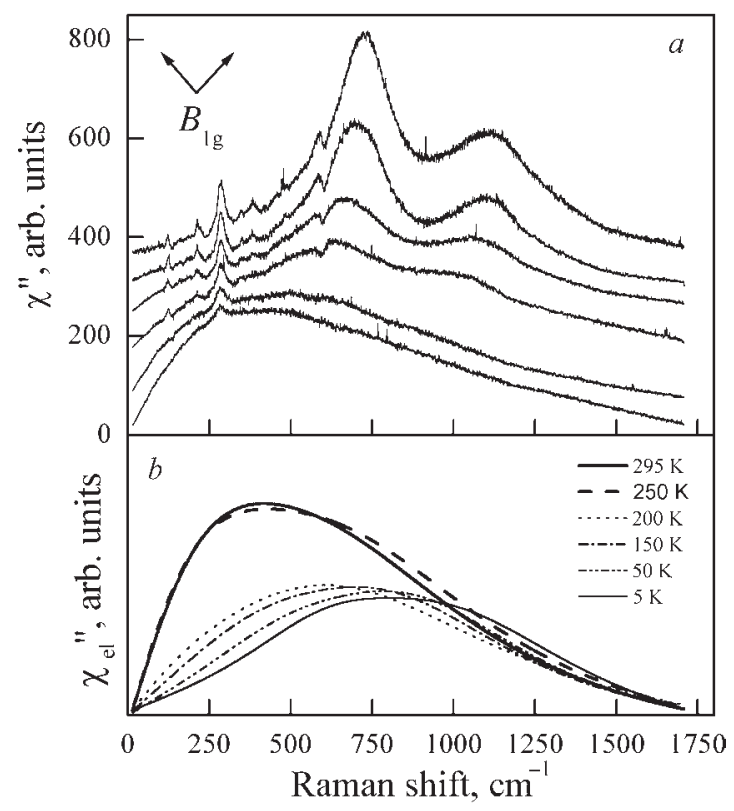

Fig. 4. Experimental $B_{1 g}$ Raman response of $\mathrm{La}_{5}{ }_{3} \mathrm{Sr}_{1} /{ }_{3} \mathrm{NiO}_{4}$ at different temperatures $(T=5,50$, $150,200,250$, and $295 \mathrm{~K}$ from top to bottom). The spectra are plotted on the same scale but are displaced vertically for clarity $(a)$. Electronic $A_{1 g}$ Raman response at the same temperatures evaluated from the experimental spectra $(b)$.

tion of the charge-transfer peak for the sample with $x=0.225$ was estimated as $\sim 840 \mathrm{~cm}^{-1}$ at $T=5 \mathrm{~K}$. The same charge gap value was obtained from the optical conductivity spectra [11]. For the sample with $x=1 / 3$, a surprisingly lower position $\left(\sim 900 \mathrm{~cm}^{-1}\right)$ of the charge transfer peak was observed in our Raman experiments in comparison with the value of $2090 \mathrm{~cm}^{-1}$ for the charge gap from the optical conductivity measurements [25].

Summarizing the comparison of our results with the theoretical calculations [23], one may to conclude that the $B_{1 g}$ response for both measured samples below $T_{c o}$ has a line shape that is closer to the pseudogap phase than to the strong insulator phase. Concerning the temperature behavior, the decreasing of the spectral weight and the shift of the peak position to a higher energy with a decreasing temperature are contrary to the theoretical predictions. It seems that the inclusion of a strong interaction between electrons and spin fluctuations [26] or the scattering of electrons on extended impurities [27] into the theory could resolve this discrepancy.

Additional information on charge dynamics can be obtained from the slope of $\chi^{\prime \prime}(\omega)$ at vanishing frequencies. This slope can be denoted as $\Gamma_{\lambda}=$ $=\lim _{\tau \rightarrow 0} \omega / \chi_{\lambda}^{\prime \prime}(\omega)$, where $\lambda=B_{1 g}$ or $B_{2 g}$. As is clearly seen in Fig. 5, the slope of the low-energy continua 


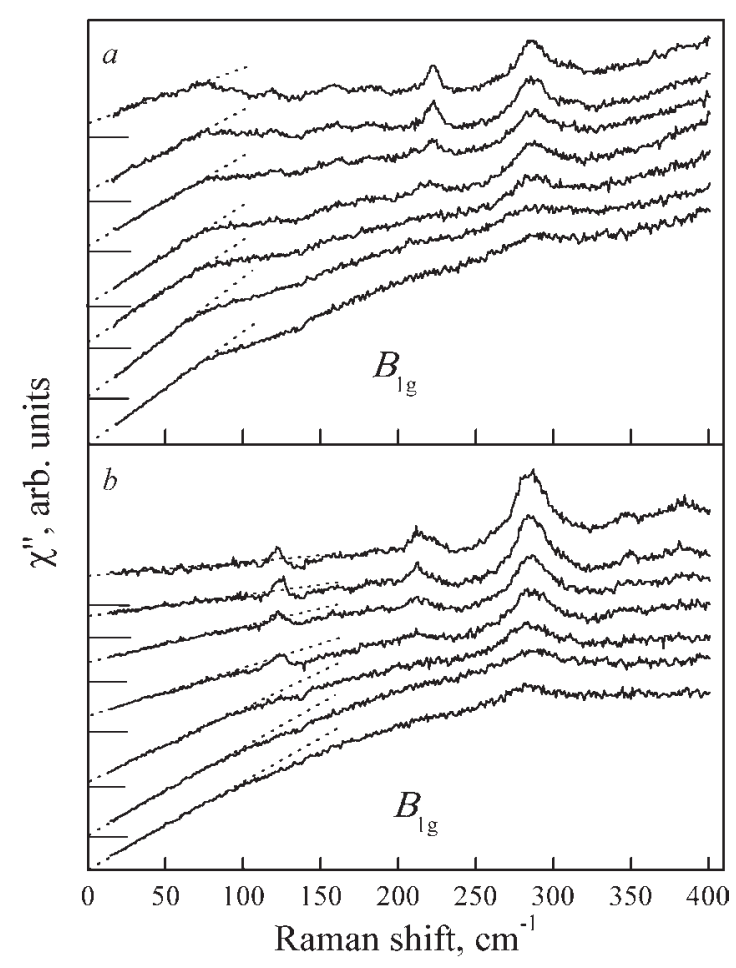

Fig. 5. Low-frequency $B_{1 g}$ Raman response of $\mathrm{La}_{1.775} \mathrm{Sr}_{0.225} \mathrm{NiO}_{4}(a)$ and $\mathrm{La}_{5}{ }_{3} \mathrm{Sr}_{1 / 3} \mathrm{NiO}_{4}(b)$ single crystals obtained at temperatures 5, 50, 100, 150, 200, 250 , and $295 \mathrm{~K}$ (from top to bottom). The dashed lines in the figures represent the slope of $\chi^{\prime \prime}(\omega)$ as $\omega \rightarrow 0$.

for both samples changes with decreasing temperature. The inverse Raman slope characterizes the quasiparticle lifetime at regions of the Fermi surface selected by the light polarization orientations. In earlier publications [23,28] it was shown that the inverse slope $1 / \Gamma_{\lambda}$ shows qualitatively different behavior for different doping regimes of various cuprate materials. At $B_{1 g}$ symmetry a strong doping dependence of the inverse slope was observed [28]. In Fig. 6, $a$ we plot the inverse slope of the Raman response obtained from the $B_{1 g}$ experimental spectra. The variation of the inverse slope with temperature for the $x=0.225$ sample clearly shows the pseudogap phase behavior, while for the $x=1 / 3$ sample the low-temperature inverse slope increases dramatically, as is characteristic for a more insulating system.

We turn now to the temperature dependence of the $B_{2 g}$ response in our samples. Our interest is connected with the attempt to observe a pseudogap in the RS spectra. The term pseudogap denotes a partial gap. An example of such a partial gap would be a situation where, within the band theory

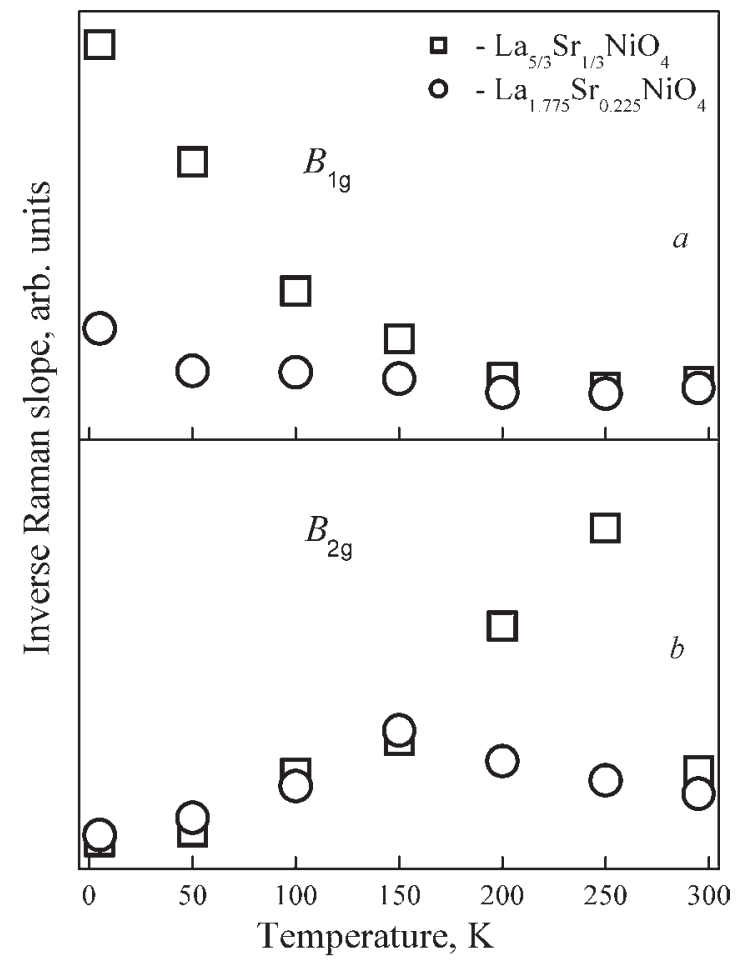

Fig. 6. Inverse slope of the $B_{1 g}(a)$ and $B_{2 g}(b)$. Raman response obtained from the experimental spectra of $\mathrm{La}_{1.775} \mathrm{Sr}_{0.225} \mathrm{NiO}_{4}$ and $\mathrm{La}_{5}{ }_{3} \mathrm{Sr}_{1 / 3} \mathrm{NiO}_{4}$ single crystals.

approximation, some regions of the Fermi surface become gapped while other parts retain their conducting properties [29]. A number of families of high-temperature cuprate oxides demonstrate evidence of the presence of a pseudogap in the normal state. As was convincingly proved in Ref. 30, the pseudogap is a signature of the electronic interactions above $T_{\mathrm{c}}$ and is not directly related to the superconducting pairing correlations. In the RS experiments on high- $T_{\mathrm{c}}$ cuprates the pseudogap state is characterized by a loss of spectral weight in the frequency range between zero and approximately $800 \mathrm{~cm}^{-1}$ and is clearly seen in $B_{2 g}$ symmetry in underdoped materials. The change of the spectra in the pseudogap state becomes very small for higher doping levels.

Figure 7 shows the $B_{2 g}$ Raman response in $\mathrm{La}_{2-x} \mathrm{Sr}_{x} \mathrm{NiO}_{4}$ at two doping levels, obtained at temperatures of $295 \mathrm{~K}$ and $5 \mathrm{~K}$. The loss of spectral weight in the low-frequency region on cooling is seen. To make things more quantitative we carried out measurements at different temperatures, and the results are shown in Figs. 8 and 9. For clarity the two temperature ranges are plotted separately. Above the charge-ordering temperature no intensity 


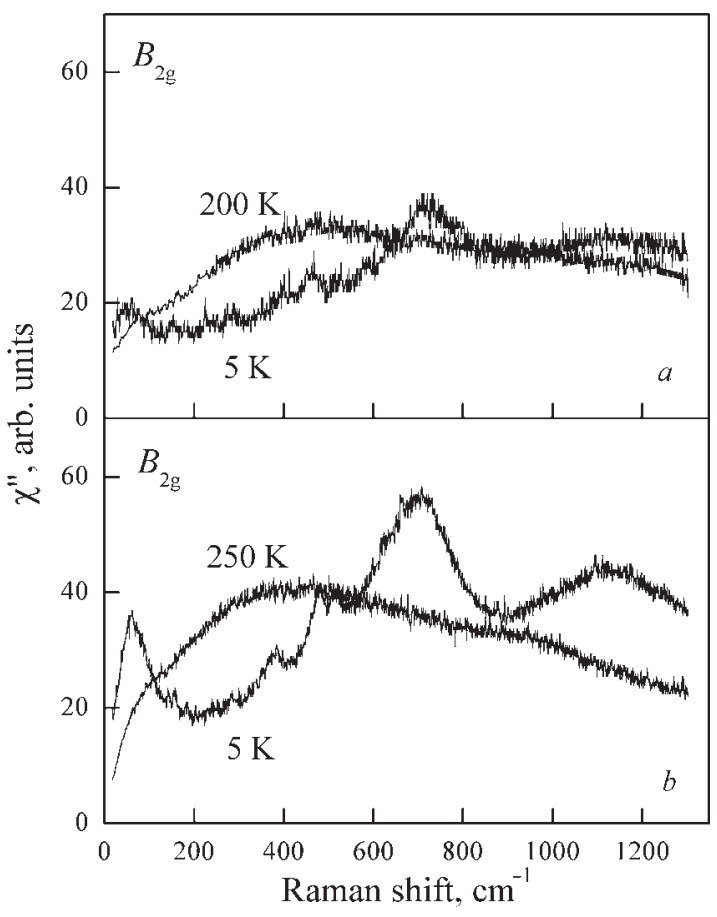

Fig. 7. Experimental $B_{2 g}$ Raman response of $\mathrm{La}_{1.775} \mathrm{Sr}_{0.225} \mathrm{NiO}_{4}(a)$ and $\mathrm{La}_{5}{ }_{3} \mathrm{Sr}_{1 / 3} \mathrm{NiO}_{4}$ (b) single crystals measured at temperatures above and below charge-ordering temperature in the frequency region $0-$ $1300 \mathrm{~cm}^{-1}$.

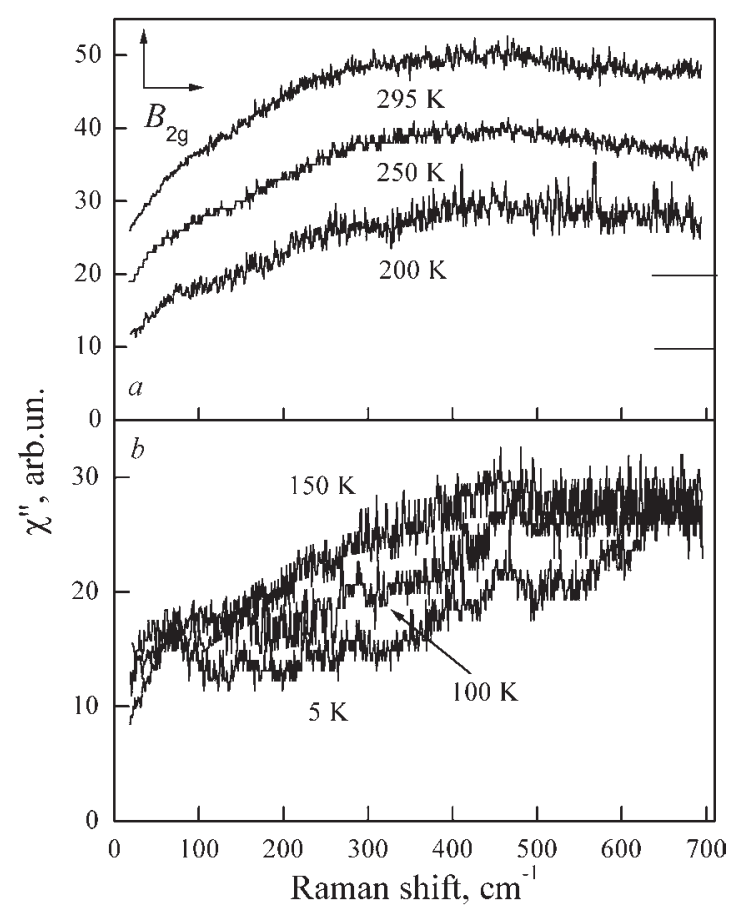

Fig. 8. $B_{2 g}$ spectra for $\mathrm{La}_{1.775} \mathrm{Sr}_{0.225} \mathrm{NiO}_{4}$. The upper panel shows spectra at $T>T_{\text {co }}(a)$. The spectra are displaced for clarity and their zeros are indicated by a tick on the vertical axis. The lower panel shows spectra at $T<T_{\text {co }}(b)$.

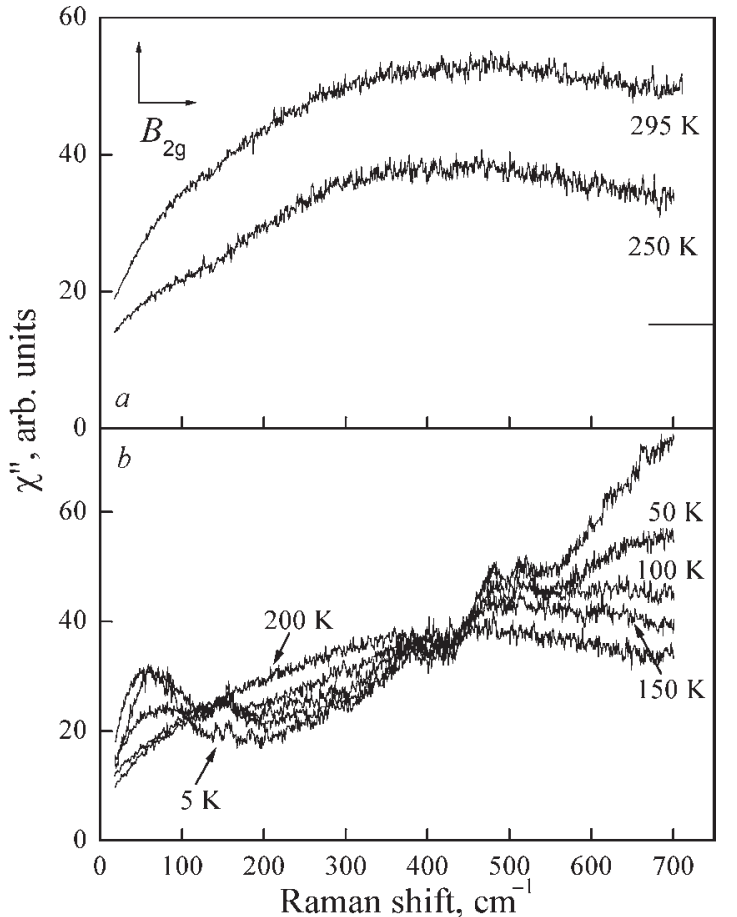

Fig. 9. $B_{2 g}$ spectra for $\mathrm{La}_{5 / 3} \mathrm{Sr}_{1 / 3} \mathrm{NiO}_{4}$. The upper panel shows spectra at $T>T_{C o}(a)$. The spectra are displaced for clarity and zero for the spectra at $295 \mathrm{~K}$ is indicated by a tick on the vertical axis. The low panel shows spectra at $T<T_{c o}(b)$.

anomalies occur (Figs. 8, $a, 9, a$ ). For $T<T_{c o}$ spectral weight is lost in the low-frequency region. In Fig. 10 we plot the difference $\Delta \chi^{\prime \prime}(\omega, T)=\chi^{\prime \prime}(\omega, T)-$ $-\chi^{\prime \prime}\left(\omega, T>T_{c o}\right)$ between the spectra measured at different temperatures below $T_{c o}$ and the spectrum obtained just above $T_{c o}$. We observe the maximal amplitude of the spectral change at approximately $300 \mathrm{~cm}^{-1}(x=0.225)$ and $250 \mathrm{~cm}^{-1}(x=1 / 3)$ (Figs. 8,b, 9,b). However, for a detailed analysis of

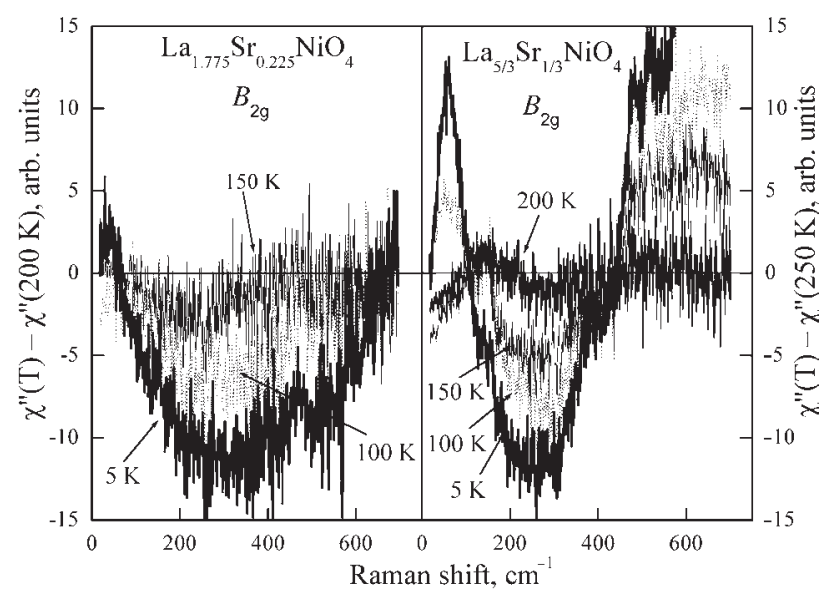

Fig. 10. The pseudogap as a function of temperature. Shown in the figure are differences between the response functions at $T<T_{c o}$ and response function just above $T_{c o}$. 


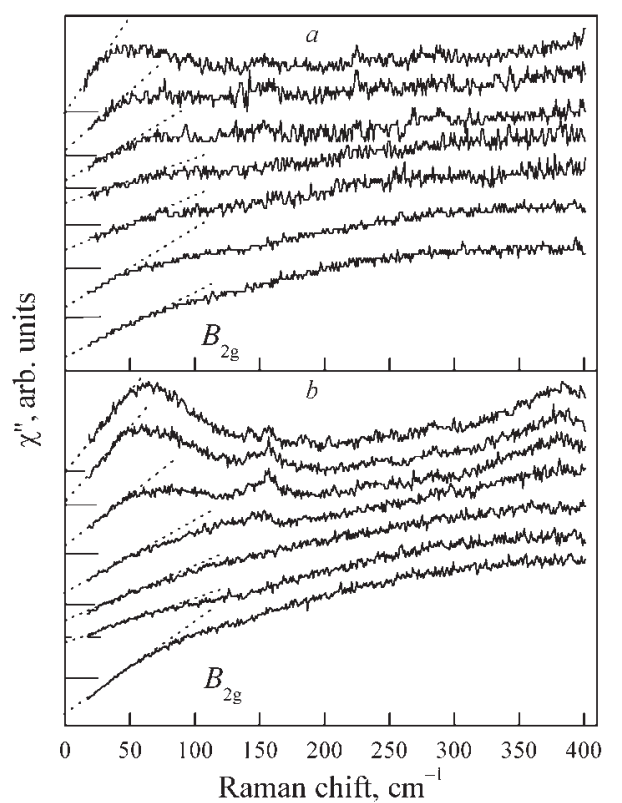

Fig. 11. Low-frequency $B_{2 g}$ Raman response of $\mathrm{La}_{1.775} \mathrm{Sr}_{0.225} \mathrm{NiO}_{4}(a)$ and $\mathrm{La}_{5}{ }_{3} \mathrm{Sr}_{1 / 3} \mathrm{NiO}_{4}$ (b) single crystals obtained at temperatures 5, 50, 100, 150, 200, 250 , and $295 \mathrm{~K}$ (from top to bottom). The dashed lines in the figures represent the slope of $\chi^{\prime \prime}(\omega)$ as $\omega \rightarrow 0$.

the pseudogap state it would be more physical to relate the «normal» and «pseudogap» spectra at the same temperature as was done in Ref. 30, where the «normal» spectra at the respective temperatures were constructed. Although the pseudogap has been observed and investigated by various methods, its interpretation is still an open issue at present. In Refs. 30,31 it was speculated that as the energy scale of the pseudogap is comparable to that of the exchange interaction $J$, the driving force is magnetic.

While the $B_{1 g}$ data cannot be linked to ordinary transport, the inverse slope observed in the $B_{2 g}$ channel was found to track the temperature dependence of the dc resistivity $[28,30]$. Moreover, the $B_{2 g}$ response is expected to be sensitive to properties of charge stripes running along the diagonal directions between in-plane $\mathrm{Ni}-\mathrm{O}$ bonds. In Fig. 11 the low-frequency $B_{2 g}$ spectra at different temperatures are shown. It is seen that the slope of the Raman response for both samples is temperature dependent. The temperature dependences of the inverse slope in the $B_{2 g}$ channel are presented in Fig. 6,b, which indicates that below $T_{c o}, 1 / \Gamma_{\lambda}$ decreases with decreasing temperature for both samples. Such metal-like behavior of the inverse $\mathrm{Ra}^{-}$ man slope demonstrates the existence of finite conductivity within the charge stripes. A crossover from two-dimensional to one-dimensional transport behavior due to the formation of stripes was pro- posed by Moshalkov et al. [32]. Direct evidence for one-dimensional transport in the stripe-ordered phase was demonstrated in Hall coefficient measurements on neodymium-doped lanthanum strontium cuprate [33].

In summary, channel-dependent Raman scattering measurements of $\mathrm{La}_{2-x} \mathrm{Sr}_{x} \mathrm{NiO}_{4} \quad(x=0.225$, $1 / 3)$ samples were carried out over a wide range of temperatures. It was found that the scattering on charge carriers for both samples is quite similar. For $A_{1 g}, B_{1 g}$, and $B_{2 g}$ symmetries a temperature-dependent redistribution of the electronic continua was observed for both compounds.

In the $B_{1 g}$ channel a crossover from the weakly interacting to pseudogap-like behavior of the electronic continua was found at the charge-ordering temperature. From the low-frequency $B_{1 g}$ spectra we have estimated the $B_{1 q}$ inverse Raman slope. Its temperature behavior is in agreement with the theoretical one for the pseudogap phase.

The pseudogap state that is characterized by a loss of spectral weight in the low-frequency region was directly observed in the $B_{2 g}$ symmetry below $T_{c o}$. The decrease of the inverse Raman slope below $T_{c o}$ and its metal-like behavior provides evidence for one-dimensional finite conductivity within the charge stripes.

We are grateful to Prof. V.V. Eremenko for permanent interest and support of our activity and for useful discussions. This work was supported by the NATO Science Programme under Grant No PST.CLG 977766.

1. S. M. Hayden, G. H. Lander, J. Zarestky, P. J. Brown, C. Stassis, P. Metcalf, and J. M. Honig, Phys. Rev. Lett. 68, 1061 (1992).

2. C. H. Chen, S.-W. Cheong, and A. S. Cooper, Phys. Rev. Lett. 71, 2461 (1993).

3. S.-W. Cheong, H. Y. Hwang, C. H. Chen, B. Battlogg, L. W. Rupp, Jr., and S. A. Carter, Phys. Rev. B49, 7088 (1994).

4. J. M. Tranquada, D. J. Buttrey, V. Sachan, and J. E. Lorenzo, Phys. Rev. Lett. 73, 1003 (1994).

5. J. M. Tranquada, J. E. Lorenzo, D. J. Buttrey, and V. Sachan, Phys. Rev. B52, 3581 (1995).

6. V. Sachan, D. J. Buttrey, J. M. Tranquada, J. E. Lorenzo, and G. Shirane, Phys. Rev. B51, 12742 (1995).

7. J. M. Tranquada, D. J. Buttrey, and V. Sachan, Phys. Rev. B54, 12318 (1996).

8. H. Yoshizawa, T. Kakeshita, R. Kajimoto, T. Tanabe, T. Katsufuji, and Y. Tokura, Phys. Rev. B61, R854 (2000).

9. H. Eskes, O. Y. Osman, R. Grimberg, W. van Saarloos, and J. Zaanen, Phys. Rev. B58, 6963 (1998). 
10. S. A. Kivelson, E. Fradkin, and V. J. Emeri, $N a^{-}$ ture (London) 393, 550 (1998).

11. Yu. Pashkevich, V. A. Blinkin, V. P. Gnezdilov, V. V. Tsapenko, V. V. Eremenko, P. Lemmens, M. Fisher, M. Grove, G. Guntherodt, L. Degiorgi, P. Wachter, and D. J. Buttrey, Phys. Rev. Lett. 84, 3919 (2000).

12. S.-H.Lee and S.-W.Cheong, Phys. Rev. Lett. 79, 2514 (1997).

13. R. J. Cava, B. Batlogg, T. T. Palstra, J. J. Krajewski, W. F. Peck, A. P. Ramirez, and L. W. Rupp, Phys. Rev. B43, 1229 (1991).

14. J. H. Jung, D.-W. Kim, T. W. Noh, H. C. Kim, H.-C. Ri, S. J. Levett, M. R. Lees, D. McK. Paul, and G. Balakrishnan, Phys. Rev. B64, 165106 (2001).

15. T. P. Devereaux, D. Einzel, B. Stadlober, R. Hackl, D. H. Leach, and J. J. Neumeier, Phys. Rev. Lett. 72, 396 (1994).

16. T. P. Devereaux D. Einzel, B. Stadlober, R. Hackl, D. H. Leach, and J. J. Neumeier, Phys. Rev. Lett. 72, 3291 (1994).

17. J. G. Naeni, X. K. Chen, K. C. Hewitt, J. C. Irwin, T. P. Devereaux, M. Okuya, T. Kimura, and K. Kishio, Phys. Rev. B57, R11077 (1998).

18. T. P. Devereaux and A. P. Kampf, Phys. Rev. B59, 6411 (1999).

19. T. P. Devereaux and A. P. Kampf, Phys. Rev. B61, 1490 (2000).

20. G. Blumberg, M. W. Klein, and S.-W. Cheong, Phys. Rev. Lett. 80, 564 (1998).
21. K. Yamamoto, T. Katsufuji, T. Tanabe, and Y. Tokura, Phys. Rev. Lett. 80, 1493 (1998).

22. S. Sugai, K. Kitamori, S. Hosoya, and K. Yamada, J. Phys. Soc. Jpn. 67, 2992 (1998).

23. J. P. Freericks and T. P. Devereauz, Phys. Rev. B64, 125110 (2001).

24. A. V. A. V. Chubukov and D. M. Frenkel, Phys. Rev. B52, 9760 (1995).

25. T. Katsufuji, T. Tanabe, T. Ishikawa, Y. Fukuda, T. Arima, and Y. Tokure, Phys. Rev. B54, R14230 (1996).

26. F. Venturini, U. Michelucci, T. P. Devereaux, and A. P. Kcampf, Phys. Rev. B62, 15204 (2000).

27. T. P. Devereaux and A. P. Kampf, Phys. Rev. B61, 1490 (2000).

28. R. Hackl, G. Krug, R. Nemetschek, M. Opel, and B. Stadlober, Proc. SPIE 2696, 194 (1996).

29. T. Timusk and B. Statt, Rep. Prog. Phys. 62, 61 (1999).

30. M. Opel, R. Nemetschek, C. Hoffman, R. Philipp, P. F. Muller, R. Hackl, I. Tutto, A. Erb, B. Revaz, E. Walker, H. Berger, and L. Forro, Phys. Rev. B61, 9752 (2000).

31. R. Nemetschek, M. Opel, C. Hoffmann, P. F. Muller, R. Hackl, H. Berger, L. Forro, A. Erb, and E. Walker, Phys. Rev. Lett. 78, 4837 (1997).

32. V. V. Moshchalkov, L. Trappeniers, and J. Vanacken, Europhys. Lett. 46, 75 (1999).

33. T. Noda, H. Eisaki, and Shin-ichi Uchida, Science 286, 265 (1999). 